\title{
Correlation between ADC values and Gleason score in evaluation of prostate cancer: multicentre experience and review of the literature
}

\author{
Rosa Manetta ${ }^{1}$, Pierpaolo Palumbo ${ }^{2}$, Camilla Gianneramo ${ }^{2}$, Federico Bruno ${ }^{2}$, Francesco Arrigoni ${ }^{2}$, \\ Raffaele Natella ${ }^{3}$, Nicola Maggialetti ${ }^{4}$, Andrea Agostini ${ }^{5}$, Andrea Giovagnoni ${ }^{5}$, Ernesto Di Cesare ${ }^{6}$, \\ Alessandra Splendiani ${ }^{2}$, Carlo Masciocchi ${ }^{2}$, Antonio Barile ${ }^{2}$ \\ ${ }^{1}$ Division of Radiology, San Salvatore Hospital, L’Aquila, Italy; ${ }^{2}$ Department of Biotechnological and Applied Clinical Sciences, University of \\ L'Aquila, L’Aquila, Italy; ${ }^{3}$ Radiology Department, University of Campania "Luigi Vanvitelli", Naples, Italy; ${ }^{4}$ Department of Life and Health "V. \\ Tiberio", University of Molise, Campobasso, Italy; ${ }^{5}$ Department of Radiology, Ospedale Riuniti, Università Politecnica delle Marche, Ancona, Italy; \\ ${ }^{6}$ Department of Life, Health and Environmental Sciences, University of L'Aquila, L'Aquila, Italy \\ Contributions: (I) Conception and design: All authors; (II) Administrative support: All authors; (III) Provision of study materials or patients: All \\ authors; (IV) Collection and assembly of data: All authors; (V) Data analysis and interpretation: All authors; (VI) Manuscript writing: All authors; (VII) \\ Final approval of manuscript: All authors. \\ Correspondence to: Pierpaolo Palumbo, MD. Department of Biotechnology and Applied Clinical Sciences, University of L'Aquila, Via Vetoio, 1 - \\ 67100 - L’Aquila, Italy. Email: palumbopierpaolo89@gmail.com.
}

\begin{abstract}
Prostate cancer $(\mathrm{PCa})$ is one of the most common cancers in male population. Multiparametric prostate magnetic resonance imaging (mp-MRI) has assumed a primary role in the diagnosis of PCa, combining morphological and functional data. Among different sequences, functional diffusion weighted imaging (DWI) is a powerful clinical tool which provides information about tissue on a cellular level. However, there is a considerable overlap between either BPH (Benign Prostate Hypertrophy) and prostatic cancer condition, as a different DWI signal intensity could be shown in the normal architecture gland. Apparent diffusion coefficient (ADC) has shown an increasing accuracy in addition to the DWI analysis in detection and localization of PCa. Notably, ADC maps derived DWI sequences has shown an overall high correlation with Gleason score (GS), considering the importance of an accurate grading of focal lesion, as main predictor factor. Furthermore, beyond the comparative analysis with DWI, ADC values has proven to be an useful marker of tumor aggressiveness, providing quantitative information on tumor characteristics according with GS and Gleason pattern, even more strenuous data are needed in order to verify which ADC analysis is more accurate.
\end{abstract}

Keywords: Prostate cancer (PCa); diffusion weighted imaging (DWI); apparent diffusion coefficient (ADC); multiparametric magnetic resonance imaging (multiparametric MRI); Gleason

Submitted Jan 21, 2019. Accepted for publication Apr 29, 2019.

doi: $10.21037 /$ gs.2019.05.02

View this article at: http://dx.doi.org/10.21037/gs.2019.05.02

\section{Background}

Prostate cancer $(\mathrm{PCa})$ is one of the most common cancer in the male population, with bone metastases in up to $70 \%$ of cases, which often require a more aggressive treatment (1-4). The incidence of $\mathrm{PCa}$ increases with age, ranging from $34 \%$ in the fifth decade, to $70 \%$ over the 80 years (5). The increase in survival (about 99\%) (5) and the development of minimally invasive procedures in PCa treatment (6) paid attention to improving PCa detection.

Diagnostic imaging has achieved high accuracy in detect tumor and defining their characteristic and aggressiveness (7).

In this regard, MRI has assumed a primary role in the 
study of various district and pathologies, thanks to its high contrast resolution (8-17). Notably, in the urogenital pathology management, MRI has become advantageous also in guiding minimally invasive treatment technique (18-20).

These evidences are confirmed by the current role of multiparametric prostate magnetic resonance imaging (mpMRI), which is the most used instrumental method for the diagnosis of $\mathrm{PCa}$, combining morphological and functional data through which also permits accurate biopsy, increasing its diagnostic yield $(21,22)$.

However, considering the amount of data, the modified version2 of Prostate Imaging Reporting and Data System (PI-RADS), initially published by the ESUR, has been formulated in order to standardize the evaluation and reporting of the mp-MRI interpretation (23), thus providing a system of analysis capable to significantly improve the preoperative detection of PCa. This classification system foresees the use of different sequences ( $\mathrm{T} 2$-weighted sequences diffusion weighted imaging (DWI) sequences and pre-contrast and post-contrast T1-weighted sequences) which together increase the detective capacity of the magnetic resonance in recognizing $\mathrm{PCa}$.

\section{DWI}

Among different multi-parametric sequences, DWI is a powerful clinical tool which provides functional information about tissue on a cellular level (24). Diffusion-weighted sequence exploits the principle of the diffuse motions of the thermally induced free water, called "Brownian" motions, according to which the diffusive properties of tissues are directly related to the amount of free interstitial water and the degree of permeability. In MRI diffusion imaging, the contrast of imaging is based on the intensity of microscopic movements. Two gradients (diffusion gradients) are added before and after a $180^{\circ}$ pulse to make a diffusion-sensitive pulse sequence. In the case of stationary spin, the dephasing of the spin due to the first diffusion gradient is followed by a perfect rephasing by the second gradient. In case of spin in motion, the power factor correction will be incomplete, with the consequent loss of signal inside the voxel (25).

The $\mathrm{b}$ value is the measure of the strength and duration of the diffusion gradients, which determines the sensitivity of the DWI sequence in identifying the zones of increased diffusivity (26). Notably, tumor tissue tends to have less diffusivity than normal tissue due to its high cellularity (27). Indeed, the normal glandular architecture is altered in PCa where the large interstitial spaces and the glandular lumens are replaced by nests of tumor cells and fibrous stroma with a consequent reduction in the movements of free water. Therefore, high-intensity signal zone on DWI images is suggestive for clinically significant cancer. However, there is considerable overlap between either $\mathrm{BPH}$ and prostatic cancer condition, as a different DWI signal intensity could be shown in the normal architecture gland.

Transitional zone (TZ) and peripheral zone (PZ), indeed, show different structure, with abundant compact fascicles of smooth muscle in the $\mathrm{TZ}$ and prevalence of glandular tissue (about the $75 \%$ of the total amount of the gland) in the PZ. This different architecture results in different DWI signal intensity, depending on the relative amount of glandular or stromal tissue. Notably, some adenoma could be characterized by high signal intensity on diffusion-weighted MR images and low ADC value, similar to tumor (28) (Figure 1). Thus, to increase the accuracy in evaluating DWI, the evaluation should include ADC map and high b-value (23), according to the current guideline for mpMRI interpretation.

The high b-value has shown a high capability in visualize clinically significant cancer by the preservation of signal intensity only in the highly restricted area, especially for subcapsular lesion or those located at the apex or the base of the gland (23). Nowadays, there is no widely accepted "high b-value," even are often used b-value more than $1,000 \mathrm{~s} / \mathrm{mm}^{2}$, with a maximum b-value ranging from 2,000 to $3,000 \mathrm{sec} / \mathrm{mm}^{2}$ (29). However, even some authors suggest the high b-value the high sensitivity in detecting clinically significant cancer (30), the latter show a significant decrease with b-value higher than $3,200 \mathrm{sec} / \mathrm{mm}^{2}$ (0.871 to 0.800 ), considering that signal to noise-ratio decrease as the b-value increase (30):

\section{Apparent diffusion coefficient (ADC)}

The ADC has shown an increased accuracy in addition to the DWI analysis in detection and localization of $\mathrm{PCa}(31)$. ADC map is a model that expresses the signal decay with increased b-value. Its accuracy widely accepted, with a sensitivity and specificity of $82.6 \%$ and $91.3 \%$ respectively, and a positive and negative predictive value of $100 \%$, as recently shown (5).

According to the ESUR guidelines, it is advisable to use at least two b-values to obtain ADC map, with the lower at $50-100 \mathrm{sec} / \mathrm{mm}^{2}$ and the higher ranging from $800-1,000$ to $2,000 \mathrm{sec} / \mathrm{mm}^{2}$ (23). Even no evidence in literature 

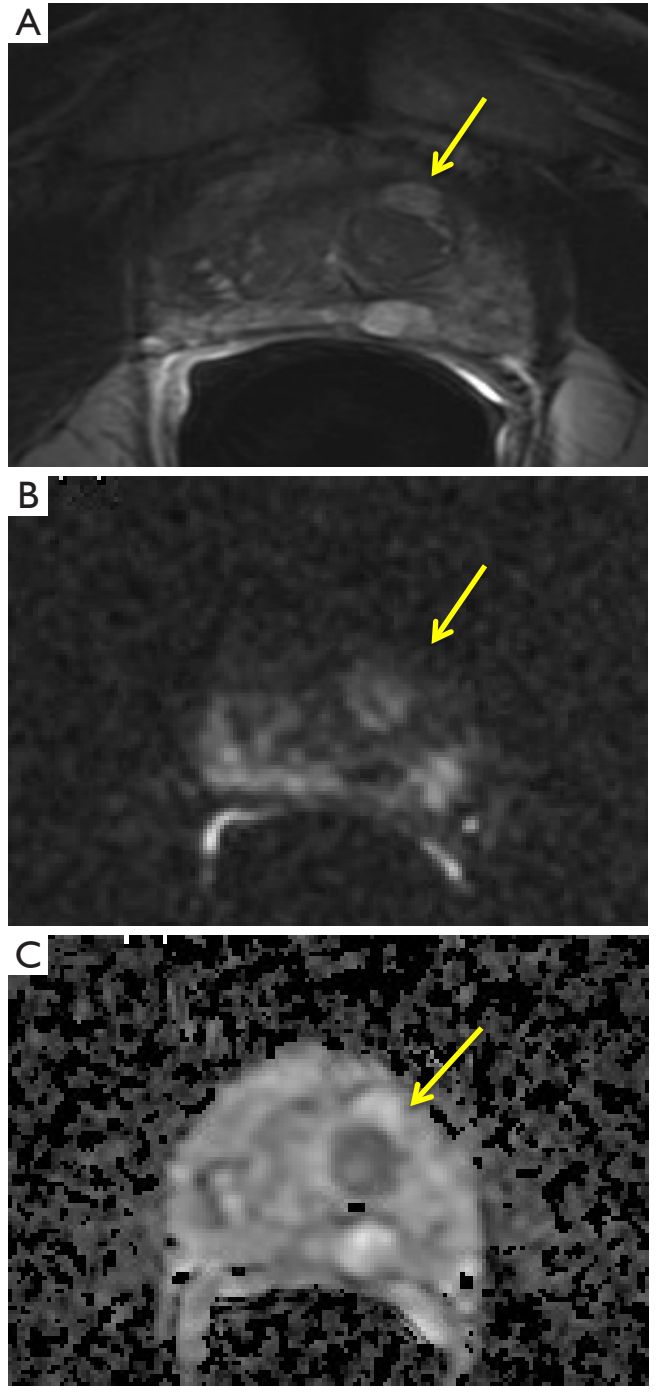

Figure 1 T2 (A), DWI (B) and ADC map (C) in healthy patient. Yellow arrows show central glandular adenoma. DWI, diffusion weighted imaging; ADC, apparent diffusion coefficient.

addressed what is the most accurate high b-value to use in ADC computation, higher b-value than $2,000 \mathrm{~s} / \mathrm{mm}^{2}$ has shown significantly lower sensitivity, as demonstrated by the accurate meta-analysis provided by Shaish et al. (32), due to the artifacts intrinsically related to the high b-value. However, ADC maps obtained with high b-value has shown high capability in the extracapsular extension of $\mathrm{PCa}$ (33).

Other attempts have been made to overcome these technical problems, as shown by Sadinski et al., who used hybrid imaging that produces maps of the changes in ADC and T2 with changing TEs (34). Sadinski et al., indeed, conclude that this approach can potentially display a
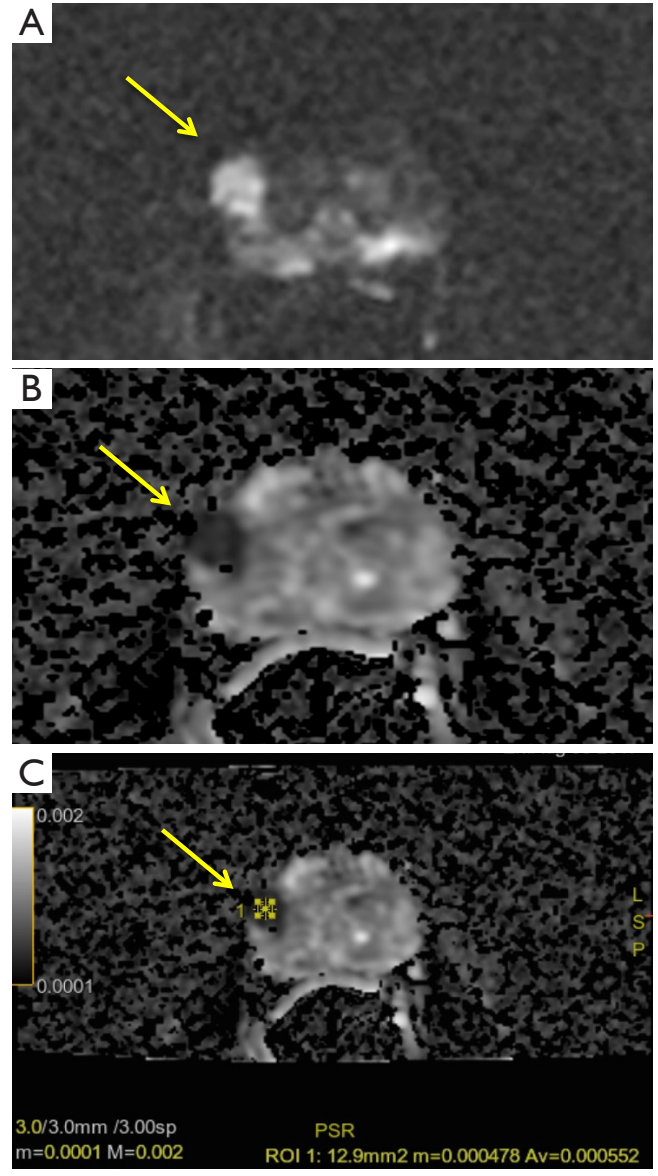

Figure 2 DWI (A) and ADC map (B) in patient with GS 8 prostate cancer (yellow arrow). ADC analysis $\left(^{*}\right)(\mathrm{C})$ show a low mean value $\left(0.552 \times 10^{-3}\right)$ consistent with the Gleason cancer grading. DWI, diffusion weighted imaging; ADC, apparent diffusion coefficient.

relatively small signal from cancerous foci within a larger normal glandular tissue. Furthermore, DWI must always be associated with the evaluation of the ADC maps also for the "T2 shine through effect", considering that the long T2-relaxation time of the glandular tissue determines a hyperintensity of $\mathrm{PZ}$ in DW images.

Beyond the comparative analysis with DWI, ADC value has proven to be a useful marker of tumor aggressiveness, providing quantitative information on tumor characteristics (24) (Figure 2). Furthermore, the assessment of local aggressiveness, through the Gleason score (GS), is the stronger predictor for localized disease. In the last few years, different studies have shown the high correlation between ADC value and GS and Gleason pattern. Indeed, biochemical recurrence rate varies considerably even in 

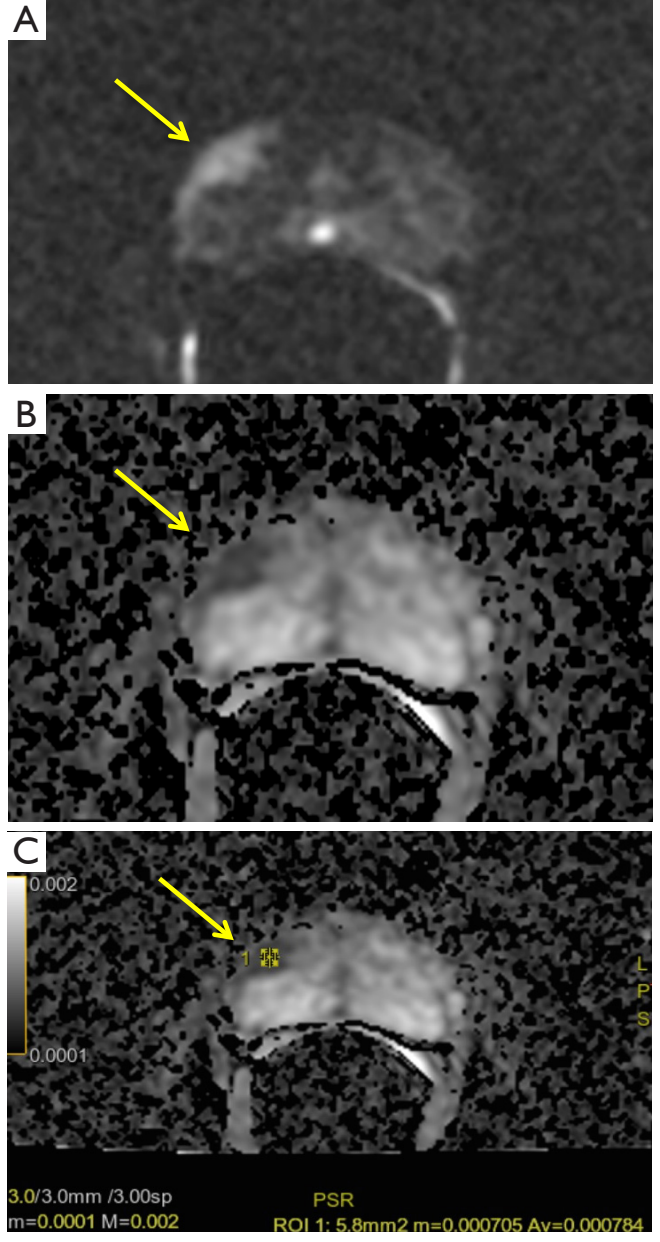

Figure 3 DWI (A) and ADC map (B) in patient with GS 7 prostate cancer (yellow arrow). ADC analysis $\left(^{*}\right)(\mathrm{C})$ show a mean value of $0.784 \times 10^{-3}$, consistent with the Gleason cancer grading. DWI, diffusion weighted imaging; ADC, apparent diffusion coefficient.

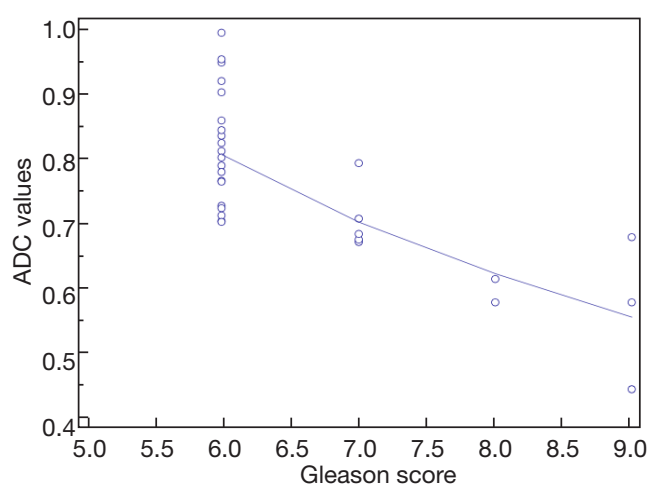

Figure 4 Correlation between ADC values and Gleason score. $\mathrm{ADC}$, apparent diffusion coefficient. the same GS 7 depending on different Gleason Pattern (GP; $3+4$ or $4+3)(31)$.

By these evidences, our experience shows similar results. Notably, we performed a retrospective analysis on 60 patients (age 45-75 years) who underwent a previous mpMRI with a PI-RADS $\geq 3 / 5$ and a subsequent histological diagnosis of GS $\geq 6$ according to the results of fusion biopsy (Magnetic resonance Imaging-Transrectal Ultrasound Image Fusion Biopsy) or radical prostatectomy. For all of them, it was analyzed the relation between $\mathrm{ADC}$ values (mean tumor ADC values) and GS (Figure 3). Analysis of data shows a characteristic distribution of value depending on $\mathrm{GS}\left(\mathrm{ADC}_{\text {mean }} \geq 0.702 \times 10^{-3} \mathrm{~mm}^{2} / \mathrm{sec}\right.$ for GS 6; $\mathrm{ADC}_{\text {mean }}$ ranging from $0.672 \times 10^{-3}$ to $0.795 \times 10^{-3} \mathrm{~mm}^{2} / \mathrm{sec}$ for GS 7 ; $\mathrm{ADC}_{\text {mean }} \leq 0.615 \times 10^{-3} \mathrm{~mm}^{2} / \mathrm{sec}$ for GS 8 or 9 and a minimum value of $\left.0.445 \times 10^{-3} \mathrm{~mm}^{2} / \mathrm{sec}\right)$, with all correlation statistically significant $(\mathrm{P}<0.0001)$ (Figure 4).

However, there is no agreed ADC tumor cut-off value that could be reliably used to determine abnormally low ADC within a lesion $(4,5)$. Nevertheless, in PI-RADS version 2, a threshold of $750-900 \mathrm{~mm}^{2} / \mathrm{s}$ is suggested as pathological ADC range value, as in our experience. It is still under debate also which is the most accurate method of measuring the $\mathrm{ADC}$ value, considering how absolute $\mathrm{ADC}$ values could depend on the selected $b$-value. Therefore, different options are under investigation beyond ADC tumor mean value, as minimum $\mathrm{ADC}$ value $\left(\mathrm{ADC}_{\min }\right)$ and normalized $\mathrm{ADC}$ value $\left(\mathrm{ADC}_{\text {ratio }}\right.$ : expressed as the ratio between tumor and non-tumor $\mathrm{ADC}$ values). $\mathrm{ADC}_{\mathrm{min}}$, indeed, is still a valid option as showing in astrocytic brain tumor, with a significant correspondence between lower $\mathrm{ADC}$ value and highest cellularity zone, thus improving the tumor grading (35).

$\mathrm{ADC}_{\text {ratio}}$, on the other hand, is relatively independent of the b-value used (31). Due to its intrinsic properties, $\mathrm{ADC}_{\text {ratio }}$ seems to be a more useful and reproducible method (36-38). In fact, among different option, even both $\mathrm{ADC}_{\text {min }}$ and $\mathrm{ADC}_{\text {ratio }}$ show a significant correlation with GS in the $\mathrm{PZ}$, only $\mathrm{ADC}_{\text {ratio }}$ show a significant capability in detecting a clinically significant tumor in $\mathrm{TZ}$ tumors, as shown by Wu et al. (24). Moreover, many authors affirm that $\mathrm{ADC}_{\text {ratio }}$ is the only method which shows a significant capability in discriminating GP 3+4 from 4+3 PCa $(29,31,39)$.

ADC maps have shown to be particularly advantageous also in both surgery and radiotherapy follow-up.

Even a relatively low sensitivity (25-29\%) in the identification of a loco-regional tumor recurrence in 
relation to lower spatial resolution, susceptibility artifacts from surgical clips or gas within the rectum or endorectal coil, and rectal motion, addition of the DWI images and the ADC maps to the T2-weighted sequences has been proven to be useful in identifying a large group of falsely negative patients as the morphological alterations did not correspond to a restricted diffusion area (40). Moreover, ADC values could show significant change also during and after radiotherapy (hypofractionated Proton and Carbon Ion irradiation), due to tumoral cell death and modifications of the local microenvironment; therefore, a finding of $\mathrm{ADC}$ values decrease after 18 months from treatment, is suggestive of tumor residual or recurrence (41).

\section{Conclusions}

In conclusion, in the last few years, different studies investigated the correlation between ADC derived DWI and tumor alteration. Through the evidence of literature, ADC analysis seems to show a high capability in defining the tumor aggressiveness, as in monitoring radiation therapy effect or surgical recurrence.

In accordance with the current literature, also our unpublished results confirm this strong correlation. However more studies are needed to clarify which ADC value analysis is the more efficient, even $\mathrm{ADC}_{\text {ratio }}$ seems to show the higher accuracy among different option.

\section{Acknowledgments}

None.

\section{Footnote}

Conflicts of Interest: The authors have no conflicts of interest to declare.

\section{References}

1. Barile A, Arrigoni F, Bruno F, et al. Present role and future perspectives of interventional radiology in the treatment of painful bone lesions. Future Oncol 2018;14:2945-55.

2. Cazzato RL, Garnon J, Ramamurthy N, et al.

Percutaneous image-guided cryoablation: current applications and results in the oncologic field. Med Oncol 2016;33:140.

3. Cazzato RL, Arrigoni F, Boatta E, et al. Percutaneous management of bone metastases: state of the art, interventional strategies and joint position statement of the Italian College of MSK Radiology (ICoMSKR) and the Italian College of Interventional Radiology (ICIR). Radiol Med 2019;124:34-49.

4. Barile A, Arrigoni F, Zugaro L, et al. Minimally invasive treatments of painful bone lesions: state of the art. Med Oncol 2017;34:53.

5. Dola EF, Nakhla OL, Genidi EAS. Assessing the validity of Prostate Imaging Reporting and Data System version 2 (PI-RADS v2) scoring system in diagnosis of peripheral zone prostate cancer. Eur J Radiol Open 2017;4:19-26.

6. Vural M, Ertaş G, Onay A, et al. Conspicuity of peripheral zone prostate cancer on computed diffusion-weighted imaging: Comparison of cDWI1500, cDWI2000, and cDWI3000. Biomed Res Int 2014;2014:768291.

7. Masciocchi C, Lanni G, Conti L, et al. Soft-tissue inflammatory myofibroblastic tumors (IMTs) of the limbs: Potential and limits of diagnostic imaging. Skeletal Radiol 2012;41:643-9.

8. Barile A, Reginelli A, De Filippo M, et al. Diagnostic imaging and intervention of the musculoskeletal system: state of the art. Acta Biomed 2018;89:5-6.

9. Barile A, Lanni G, Conti L, et al. Lesions of the biceps pulley as cause of anterosuperior impingement of the shoulder in the athlete: potentials and limits of MR arthrography compared with arthroscopy. Radiol Med 2013;118:112-22.

10. Bruno F, Smaldone F, Varrassi M, et al. MRI findings in lumbar spine following O2-O3 chemiodiscolysis: A longterm follow-up. Interv Neuroradiol 2017;23:444-50.

11. Masciocchi C, Conti L, D'Orazio F, et al. Errors in musculoskeletal MRI. In: Errors in Radiology 2012:209-17.

12. Masciocchi C, Barile A, Lelli S, et al. Magnetic Resonance Imaging (MRI) and arthro-MRI in the evaluation of the chondral pathology of the knee joint. Radiol Med 2004:108:149-58.

13. Barile A, Conti L, Lanni G, et al. Evaluation of medial meniscus tears and meniscal stability: Weight-bearing MRI vs arthroscopy. Eur J Radiol 2013;82:633-9.

14. Barile A, Arrigoni F, Bruno F, et al. Computed Tomography and MR Imaging in Rheumatoid Arthritis. Radiol Clin North Am 2017;55:997-1007.

15. Michelini G, Corridore A, Torlone S, et al. Dynamic MRI in the evaluation of the spine: State of the art. Acta Biomed 2018;89:89-101.

16. Splendiani A, Ferrari F, Barile A, et al. Occult neural foraminal stenosis caused by association between disc degeneration and facet joint osteoarthritis: Demonstration 
with dedicated upright MRI system. Radiol Med 2014;119:164-74.

17. Splendiani A, Perri M, Grattacaso G, et al. Magnetic resonance imaging (MRI) of the lumbar spine with dedicated G-scan machine in the upright position: a retrospective study and our experience in 10 years with 4305 patients. Radiol Med 2016;121:38-44.

18. Ferrari F, Arrigoni F, Miccoli A, et al. Effectiveness of Magnetic Resonance-guided Focused Ultrasound Surgery (MRgFUS) in the uterine adenomyosis treatment: technical approach and MRI evaluation. Radiol Med 2016;121:153-61.

19. Mascaretti S, Ferrari F, Miccoli A, et al. Feasibility of magnetic resonance-guided focus ultrasound surgery (MRgFUS) in the uterine fibroid treatment : evaluation of the treatment radicalization in single and multiple fibroids correlated to clinical outcome. Clin Exp Obstet Gynecol 2018;45:84-7.

20. Masciocchi C, Arrigoni F, Ferrari F, et al. Uterine fibroid therapy using interventional radiology mini-invasive treatments: current perspective. Med Oncol 2017;34:52.

21. Del Monte M, Leonardo C, Salvo V, et al. MRI/US fusion-guided biopsy: performing exclusively targeted biopsies for the early detection of prostate cancer. Radiol Med 2018;123:227-34.

22. Faiella E, Santucci D, Greco F, et al. Analysis of histological findings obtained combining US/mp-MRI fusion-guided biopsies with systematic US biopsies: mpMRI role in prostate cancer detection and false negative. Radiol Med 2018;123:143-52.

23. Weinreb JC, Barentsz JO, Choyke PL, et al. PI-RADS Prostate Imaging - Reporting and Data System: 2015, Version 2. Eur Urol 2016;69:16-40.

24. Wu X, Reinkainen P, Vanhanen A, et al. Correlation between apparent diffusion coefficient value on diffusionweighted MR imaging and Gleason score in prostate cancer. Diagn Interv Imaging 2017;98:63-71.

25. Brunsing RL, Schenker-Ahmed NM, White NS, et al. Restriction spectrum imaging: An evolving imaging biomarker in prostate MRI. J Magn Reson Imaging 2017;45:323-36.

26. Xi Y, Liu A, Olumba F, et al. Low-to-high b-value DWI ratio approaches in multiparametric MRI of the prostate: feasibility, optimal combination of b-values, and comparison with ADC maps for the visual presentation of prostate cancer. Quant Imaging Med Surg 2018;8:557-67.

27. White NS, McDonald C, Farid N, et al. Diffusionweighted imaging in cancer: physical foundations and applications of restriction spectrum imaging. Cancer Res 2014;74:4638-52.

28. Barentsz JO, Richenberg J, Clements R, et al. ESUR prostate MR guidelines 2012. Eur Radiol 2012;22:746-57.

29. Tamada T, Prabhu V, Li J, et al. Assessment of prostate cancer aggressiveness using apparent diffusion coefficient values: impact of patient race and age. Abdom Radiol (NY) 2017;42:1744-51.

30. Feng Z, Min X, Margolis DJA, et al. Evaluation of different mathematical models and different $b$-value ranges of diffusion-weighted imaging in peripheral zone prostate cancer detection using b-value up to $4500 \mathrm{~s} / \mathrm{mm} 2$. PLoS One 2017;12:e0172127.

31. Alessandrino F, Taghipour M, Hassanzadeh E, et al. Predictive role of PI-RADSv2 and ADC parameters in differentiating Gleason pattern $3+4$ and $4+3$ prostate cancer. Abdom Radiol (NY) 2019;44:279-85.

32. Shaish H, Kang SK, Rosenkrantz AB. The utility of quantitative $\mathrm{ADC}$ values for differentiating high-risk from low-risk prostate cancer: a systematic review and metaanalysis. Abdom Radiol (NY) 2017;42:260-70.

33. Kido A, Tamada T, Sone T, et al. Incremental value of high $\mathrm{b}$ value diffusion-weighted magnetic resonance imaging at 3-T for prediction of extracapsular extension in patients with prostate cancer: preliminary experience. Radiol Med 2017;122:228-38.

34. Sadinski M, Karczmar G, Peng Y, et al. Pilot Study of the Use of Hybrid Multidimensional T2-Weighted ImagingDWI for the Diagnosis of Prostate Cancer and Evaluation of Gleason Score. AJR Am J Roentgenol 2016;207:592-8.

35. Kitis O, Altay H, Calli C, et al. Minimum apparent diffusion coefficients in the evaluation of brain tumors. Eur J Radiol 2005;55:393-400.

36. Boesen L, Chabanova E, Løgager V, et al. Apparent diffusion coefficient ratio correlates significantly with prostate cancer gleason score at final pathology. J Magn Reson Imaging 2015;42:446-53.

37. Lebovici A, Sfrangeu SA, Feier D, et al. Evaluation of the normal-to-diseased apparent diffusion coefficient ratio as an indicator of prostate cancer aggressiveness. BMC Med Imaging 2014;14:15.

38. De Cobelli F, Ravelli S, Esposito A, et al. Apparent diffusion coefficient value and ratio as noninvasive potential biomarkers to predict prostate cancer grading: Comparison with prostate biopsy and radical prostatectomy specimen. AJR Am J Roentgenol 2015;204:550-7.

39. Hötker AM, Mazaheri Y, Aras Ö, et al. Assessment of prostate cancer aggressiveness by use of the combination 
of quantitative DWI and dynamic contrast-enhanced MRI. AJR Am J Roentgenol 2016;206:756-63.

40. Kitajima K, Hartman RP, Froemming AT, et al. Detection of local recurrence of prostate cancer after radical prostatectomy using endorectal coil MRI at 3 T: Addition of DWI and dynamic contrast enhancement to T2-

Cite this article as: Manetta R, Palumbo P, Gianneramo C, Bruno F, Arrigoni F, Natella R, Maggialetti N, Agostini A, Giovagnoni A, Di Cesare E, Splendiani A, Masciocchi C, Barile A. Correlation between ADC values and Gleason score in evaluation of prostate cancer: multicentre experience and review of the literature. Gland Surg 2019;8(Suppl 3):S216-S222. doi: $10.21037 / g s .2019 .05 .02$ weighted MRI. AJR Am J Roentgenol 2015;205:807-16. 41. Wolf MB, Edler C, Tichy D, et al. Diffusion-weighted MRI treatment monitoring of primary hypofractionated proton and carbon ion prostate cancer irradiation using raster scan technique. J Magn Reson Imaging 2017;46:850-60. 